\title{
ON CONTACT STRUCTURE OF HYPERSURFACES IN COMPLEX MANIFOLDS, II
}

\author{
YOSHIHIRO TASHIRO
}

(Received February 2,1963)

A previous paper with the same title will be cited as Paper I, and we continue to use terminologies in it. As the objects of the present paper are almost contact manifolds, let us denote a $(2 p-1)$-dimensional manifold by $M$ and a local coordinate system in $M$ by $\left(y^{h}\right)$.

Given an almost Grayan manifold $M$ with almost contact c-tensor $f$ and its associated metric tensor $g$, we constructed an almost Hermitian manifold $\widetilde{M}$ as follows: The underlying manifold of $\widetilde{M}$ is the product of $M$ with a straight line $I=(-\infty,+\infty)$, the almost complex structure $F$ is defined by the natural extension of $f$ into $\widetilde{M}$, and the metric tensor $G$ by

$$
G=\left(G_{\mu \lambda}\right)=e^{-2 t} \tilde{g}=e^{-2 t}\left(\begin{array}{ll}
g_{j i} & 0 \\
0 & 1
\end{array}\right), \quad t \in I .
$$

The manifold $M$ is identified with the hypersurface $M \times 0$, which is totally umbilical in $\widetilde{M}$. A coordinate system in $\widetilde{M}$ such as $\left(y^{h}, t\right)$ is called an adapted one.

Moreover we saw that, if $M$ is Grayan, almost Sasakian or Sasakian, then the manifold $\widetilde{M}$ constructed above is Hermitian, almost Kählerian or Kählerian respectively, and vice versa. In this sense, the almost Grayan manifold $\widetilde{M}$ is naturally associated with $M$, and we call it the enveloping manifold of $M$. The purpose of the present paper is to study properties of manifolds with almost contact metric structure through the properties of their enveloping manifolds.

7. Contact c-tensor calculus. Since the metric tensor $G$ of the enveloping manifold $\widetilde{M}$ is conformal to $\widetilde{g}$ of the product manifold $M \times I$ with scalar factor $\rho=e^{-t}$, the Christoffel symbol of $\widetilde{M}$ is given by

$$
\left\{\begin{array}{c}
\tilde{\kappa} \\
\mu \lambda
\end{array}\right\}=\left\{\begin{array}{c}
\kappa \\
\mu \lambda
\end{array}\right\}+\delta_{\mu}^{\kappa} \rho_{\lambda}+\delta_{\lambda}^{\kappa} \rho_{\mu}-g_{\mu \lambda} \rho^{\kappa},
$$


where $\left\{\begin{array}{c}\kappa \\ \mu \lambda\end{array}\right\}$ is the Christoffel symbol of $M \times I$, and $\rho_{\lambda}=\partial_{\lambda} \log \rho_{\text {. }}$. Since we have

$$
\rho_{i}=\rho^{h}=0, \rho_{\infty}=\rho^{\infty}=-1
$$

in an adapted coordinate system, the non-trivial components of $\left\{\begin{array}{c}\tilde{\kappa} \\ \mu \lambda\end{array}\right\}$ are there

$$
\left\{\begin{array}{c}
\widetilde{h} \\
j i
\end{array}\right\}=\left\{\begin{array}{c}
h \\
j i
\end{array}\right\},\left\{\begin{array}{c}
\widetilde{\infty} \\
j i
\end{array}\right\}=g_{j i},\left\{\begin{array}{c}
\widetilde{h} \\
j \infty
\end{array}\right\}=-\delta_{j}^{h},\left\{\begin{array}{c}
\tilde{\infty} \\
\infty \infty
\end{array}\right\}=-1 .
$$

The curvature tensor $\widetilde{K}_{v \mu \lambda}{ }^{\kappa}$ of $\widetilde{M}$ is given by

$$
\widetilde{K}_{\nu \mu \lambda}{ }^{\kappa}=K_{\nu \mu \lambda}{ }^{\kappa}-\delta_{v}^{\kappa} \rho_{\mu \lambda}+\delta_{\mu}^{\kappa} \rho_{\nu \lambda}-g_{\mu \lambda} \rho_{\nu}{ }^{\kappa}+g_{\nu \lambda} \rho_{\mu}{ }^{\kappa},
$$

where

$$
\rho_{\mu \lambda}=\nabla_{\mu} \rho_{\lambda}-\rho_{\mu} \rho_{\lambda}+\frac{1}{2} g_{\mu \lambda} \rho_{\kappa} \rho^{\kappa}, \rho_{\mu}^{\kappa}=\rho_{\mu \lambda} g^{\lambda_{\kappa}}
$$

Since we have

$$
\rho_{j i}=\frac{1}{2} g_{j i}, \rho_{\infty i}=0, \rho_{\infty \infty}=-\frac{1}{2},
$$

the non-trivial components of $\widetilde{K}_{v \mu \lambda}{ }^{\kappa}$ are only

$$
\widetilde{K}_{k j i}^{h}=K_{k j i}^{h}-\delta_{k}^{h} g_{j i}+\delta_{j}^{h} g_{k i}
$$

and those of the Ricci tensor $\widetilde{K}_{\mu \lambda}$ are only

$$
\widetilde{K}_{j i}=K_{j i}-(2 p-2) g_{j i},
$$

and the scalar curvature is given by

$$
\tilde{\kappa}=(2 p-1) e^{2 t}(\kappa-1) / 2 p .
$$

For a tensor field in $\widetilde{M}$, say $T$ having components $\left(\begin{array}{cc}T_{i}{ }^{h} & T_{i}^{\infty} \\ T_{\infty}^{h} & T_{\infty}^{\infty}\end{array}\right)$ in an adapted coordinate system, the covariant derivative $\widetilde{\nabla} T$ is separated into the components

$$
\left\{\begin{array}{l}
\widetilde{\nabla}_{j} T_{i}{ }^{h}=\nabla_{j} T_{i}{ }^{h}-\delta_{j}^{h} T_{i}^{\infty}-g_{j i} T_{\infty}{ }^{h}, \\
\widetilde{\nabla}_{j} T_{i}{ }^{\infty}=\nabla_{j} T_{i}{ }^{\infty}+g_{j h} T_{i}{ }^{h}-g_{j i} T_{\infty}{ }^{\infty}, \\
\widetilde{\nabla}_{j} T_{\infty}{ }^{h}=\nabla_{j} T_{\infty}{ }^{h}-\delta_{j}^{h} T_{\infty}{ }^{\infty}+T_{j}{ }^{h}, \\
\widetilde{\nabla}_{j} T_{\infty}{ }^{\infty}=\nabla_{j} T_{\infty}{ }^{\infty}+g_{j h} T_{\infty}{ }^{h}+T_{j}{ }^{\infty}, \\
\widetilde{\nabla}_{\infty} T_{\mu}{ }^{\lambda}=\partial_{\infty} T_{\mu}^{\lambda} .
\end{array}\right.
$$


In particular, the covariant derivative of the almost complex structure $F$ is separated into

$$
\left\{\begin{array}{l}
\widetilde{\nabla}_{j} F_{i}^{h}=\nabla_{j} f_{i}^{h}-\delta_{j}^{h} f_{i}-g_{j i} f^{h} \\
\widetilde{\nabla}_{j} F_{i}^{\infty}=\nabla_{j} f_{i}-f_{j i} \\
\widetilde{\nabla}_{j} F_{\infty}^{h}=\nabla_{j} f^{h}+f_{j}^{h} \\
\widetilde{\nabla}_{j} F_{\infty}^{\infty}=0 \\
\widetilde{\nabla}_{\infty} F_{\mu}^{\lambda}=0
\end{array}\right.
$$

and that of the covariant tensor $F_{*}$ into

$$
\left\{\begin{array}{l}
\widetilde{\nabla}_{j} F_{i h}=e^{-2 t}\left(\nabla_{j} f_{i h}+g_{j i} f_{h}-g_{j h} f_{i}\right), \\
\widetilde{\nabla}_{j} F_{i \infty}=e^{-2 t}\left(\nabla_{j} f_{i}-f_{j i}\right) \\
\widetilde{\nabla}_{j} F_{\propto \infty}=0 \\
\widetilde{\nabla}_{\infty} F_{\mu \lambda}=0 .
\end{array}\right.
$$

The Nijenhuis c-tensor $n$ of $f$ defined by (1.20) is identical with the Nijenhuis tensor of $F$ with respect to the adapted coordinate system, and we have

$$
n_{\mu \lambda}{ }^{\kappa}=N_{\mu \lambda}{ }^{\kappa}=F_{\mu}^{\alpha}\left(\widetilde{\nabla}_{\alpha} F_{\lambda}{ }^{\kappa}-\widetilde{\nabla}_{\lambda} F_{\alpha}^{\kappa}\right)-F_{\lambda}^{\alpha}\left(\widetilde{\nabla}_{\alpha} F_{\mu}{ }^{\kappa}-\widetilde{\nabla}_{\mu} F_{\alpha}{ }^{\kappa}\right)
$$

and its components are written in the form

$$
\left\{\begin{aligned}
& n_{j i}{ }^{h}=f_{j}{ }^{a}\left(\nabla_{a} f_{i}{ }^{h}-\nabla_{i} f_{a}{ }^{h}\right)-f_{i}{ }^{a}\left(\nabla_{a} f_{j}{ }^{h}-\nabla_{j} f_{a}{ }^{h}\right) \\
&-f_{j} \nabla_{i} f^{h}+f_{i} \nabla_{j} f^{h}, \\
& n_{j i}{ }^{\infty}=f_{j}{ }^{a}\left(\nabla_{a} f_{i}-\nabla_{i} f_{a}\right)-f_{i}{ }^{a}\left(\nabla_{a} f_{j}-\nabla_{j} f_{a}\right), n_{\infty i}{ }^{h}=f^{a}\left(\nabla_{a} f_{i}{ }^{h}-\nabla_{i} f_{a}{ }^{h}\right)-f_{i}{ }^{a} \nabla_{a} f^{h}, \\
& n_{\infty i}{ }^{\infty}=f^{a} \nabla_{a} f_{i} .
\end{aligned}\right.
$$

As is well known, the Nijenhuis tensor $N_{\mu \lambda}{ }^{\kappa}$ is skew symmetric in $\lambda$ and $\mu$, and hybrid in $\kappa$ and $\lambda$ :

$$
N_{\mu \lambda}{ }^{\alpha} F_{\alpha}^{\kappa}+N_{\mu \alpha}{ }^{\alpha} F_{\lambda}^{\alpha}=0,
$$

and pure in $\lambda$ and $\mu$ :

$$
N_{\mu \alpha}{ }^{\kappa} F_{\lambda}{ }^{\alpha}-N_{\alpha \lambda}{ }^{\kappa} F_{\mu}{ }^{\alpha}=0 .
$$

In an adapted coordinate system, the equation (7.15) is separated into

$$
\left\{\begin{array}{l}
n_{j i}{ }^{a} f_{a}{ }^{h}+n_{j a}{ }^{h} f_{i}^{a}+n_{j i}{ }^{\infty} f^{h}+n_{j \infty}{ }^{h} f_{i}=0, \\
n_{j i}{ }^{a} f_{a}+n_{j a}{ }^{\infty} f_{i}^{a}+n_{j \infty}{ }^{\infty} f_{i}=0, \\
n_{j \infty}{ }^{a} f_{a}{ }^{h}+n_{j \infty}{ }^{\infty} f_{i}{ }^{h}+n_{j a}{ }^{h} f^{a}=0,
\end{array}\right.
$$




$$
\left\{\begin{array}{l}
n_{j \infty}{ }^{a} f_{a}+n_{j a}{ }^{\infty} f^{a}=0, \\
n_{\infty i}{ }^{a} f^{h}+n_{i \infty}{ }^{\infty} f^{h}+n_{\infty a}{ }^{h} f_{i}{ }^{a}=0, \\
n_{\infty i}{ }^{a} f_{a}+n_{\infty a}{ }^{\infty} f_{i}^{a}=0, \\
u_{\infty a}{ }^{h} f^{a}=0, \\
n_{\infty a}{ }^{\infty} f^{a}=0,
\end{array}\right.
$$

and (7.16) into

$$
\left\{\begin{array}{l}
n_{j a}{ }^{h} f_{i}^{a}+n_{j \infty}{ }^{h} f_{i}-n_{a i}{ }^{h} f_{j}^{a}-n_{\infty i}{ }^{h} f_{j}=0, \\
n_{j a}{ }^{\infty} f_{i}^{a}+n_{j \infty}{ }^{\infty} f_{i}-n_{a i}{ }^{\infty} f_{j}^{a}-n_{\infty i}{ }^{\infty} f_{j}=0, \\
n_{j a}{ }^{h} f^{a}-n_{a \infty}{ }^{h} f_{j}^{a}=0, \\
n_{j a}{ }^{\infty} f^{a}-n_{a \infty}{ }^{\infty} f_{j}^{a}=0 .
\end{array}\right.
$$

The equation (7.16) is equivalent to

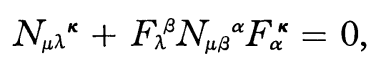

which is separated into

$$
\left\{\begin{array}{l}
n_{j i}{ }^{h}+f_{i}^{b} n_{j b}{ }^{a} f_{a}{ }^{h}+f_{i} n_{j \infty}{ }^{a} f_{a}{ }^{h}+f_{i}^{b} n_{j b}{ }^{\infty} f^{h}+f_{i} n_{j \infty}{ }^{\infty} f^{h}=0, \\
n_{j i}{ }^{\infty}+f_{i}^{b} n_{j b}{ }^{a} f_{a}+f_{i} n_{j \infty}{ }^{a} f_{a}=0, \\
n_{j \infty}{ }^{h}+f^{b} n_{j b}{ }^{a} f_{a}{ }^{h}+f^{b} n_{j b}{ }^{\infty} f^{h}=0, \\
n_{j \infty}{ }^{\infty}+f^{b} n_{j b}{ }^{a} f_{a}=0, \\
n_{\infty i}{ }^{h}+f_{i}^{b} n_{\infty b}{ }^{a} f_{a}{ }^{h}+f_{i}^{b} n_{\infty}{ }^{a} f_{a}=0, \\
n_{\infty i}{ }^{\infty}+f_{i}^{b} n_{\infty b}{ }^{a} f_{a}=0 .
\end{array}\right.
$$

The equations (7.20) can be directly derived from (7.17) and (7.18), too.

8. Special almost Grayan structures. Based on properties of almost complex structure, S.Kotô [12] made clear the relations of reduction among special almost Hermitian manifolds, and put them into the diagram

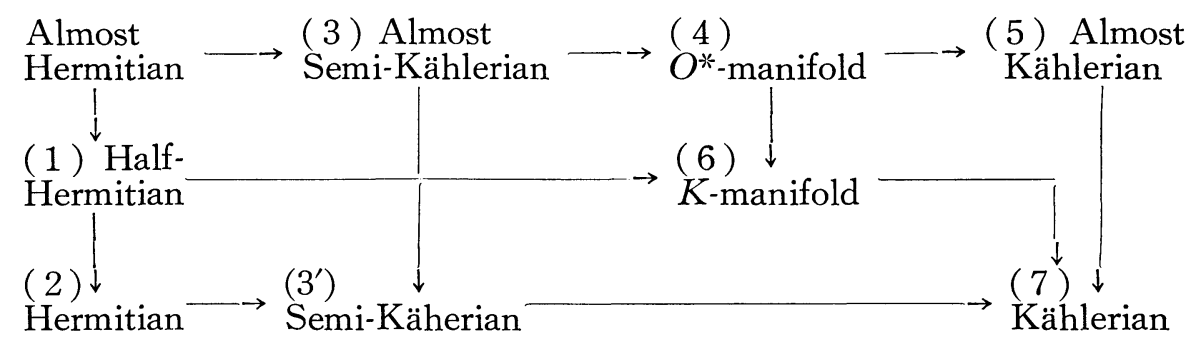

These manifolds are respectively characterized by the following properties:

(1) The covariant Nijenhuis tensor $N_{\mu \lambda_{\kappa}}$ is skew symmetric.

(2) The Nijenhuis tensor $N_{\mu \lambda}{ }^{\kappa}$ vanishes, and it is equivalent to the 
property that $\widetilde{\nabla}_{\mu} F_{\lambda}^{\kappa}$ is hybrid in $\lambda$ and $\mu$.

(3) The fundamental form $\Theta=F_{\mu \lambda} d x^{\mu} \wedge d x^{\lambda}$ is coclosed:

$$
\widetilde{\delta} \Theta=0, \widetilde{\nabla}_{\kappa} F_{\lambda}^{\kappa}=0 .
$$

(4) The covariant derivative $\widetilde{\nabla}_{\mu} F_{\lambda}{ }^{\kappa}$ is pure in $\lambda$ and $\mu$ :

$$
F_{\mu}^{\alpha} \widetilde{\nabla}_{\alpha} F_{\lambda}^{\kappa}-F_{\lambda}^{\alpha} \widetilde{\nabla}_{\mu} F_{\alpha}^{\kappa}=0 .
$$

(5) The fundamental form $\Theta$ is closed:

$$
d \Theta=0, F_{\mu \lambda \kappa}=\widetilde{\nabla}_{\mu} F_{\lambda \kappa}+\widetilde{\nabla}_{\lambda} F_{\kappa \mu}+\widetilde{\nabla}_{\kappa} F_{\mu \lambda}=0 .
$$

(6) The covariant almost complex tensor $F_{\lambda \mu}$ is a Killing tensor :

$$
\widetilde{\nabla_{\mu}} F_{\lambda_{\kappa}}+\widetilde{\nabla}_{\lambda} F_{\mu \kappa}=0 \text {. }
$$

(7) The almost complex structure $F_{\lambda}^{\kappa}$ is covariant constant:

$$
\widetilde{\nabla}_{\mu} F_{\lambda \kappa}=0 \text {. }
$$

From our view-point, to each $\widetilde{M}$ of the above manifolds corresponds a special almost Grayan manifold $M$, which has $\widetilde{M}$ as enveloping manifold. We shall give the corresponding manifolds the names at the corresponding places in the following diagram :

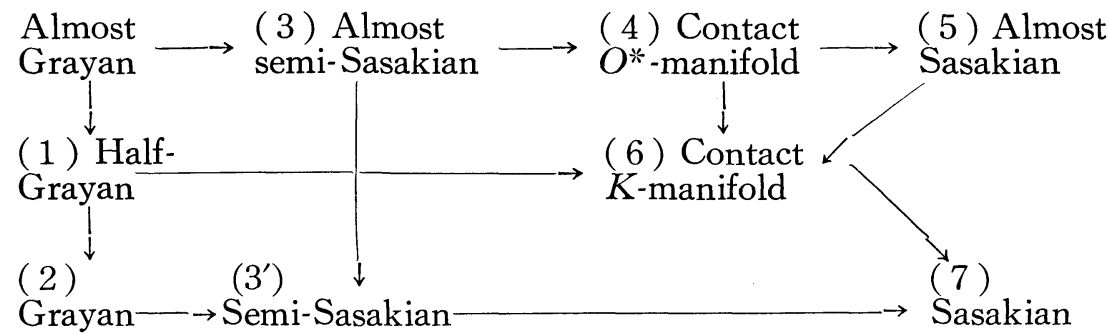

We shall see how each of the manifolds is characterized by the property of its almost contact structure. The change of the arrow issuing from (5) will be made clear in later.

(1) Half-Grayan manifold. Since the covariant Nijenhuis extensor of $f$ in $M$ is given by

$$
n_{\mu \lambda \kappa}=n_{\mu \lambda}{ }^{\alpha} g_{\alpha \kappa}=e^{2 t} N_{\mu \lambda_{\kappa}},
$$

the skew symmetry of $N_{\mu \lambda_{\kappa}}$ in all indices implies that of $n_{\mu \lambda_{\kappa}}$, in particular, that of $n_{j i h}$. Conversely, if $n_{j i h}$ is skew symmetric, then we have $n_{j \infty \infty \infty}=0$ from $(7.20)_{4}$ and $n_{j \infty}{ }^{h} f_{h}=n_{j a}{ }^{\infty} f^{a}=0$ from $(7.17)_{4,7}$. Further, from $(7.20)_{2,3}$, we have

$$
n_{j \infty h}=f^{b} n_{j b a} f_{h}{ }^{a}=-f_{h}^{b} n_{j b a} f^{a}=f_{h}^{b} n_{j b} f_{a}=-n_{j h \infty} .
$$


Thus the covariant Nijenhuis extensor $n_{\mu \lambda_{\kappa}}$ is skew symmetric in all indices, and we can state that

THEOREM 8.1. A half-Grayan manifold is characterized by the skew symmetry of the covariant tensor $n_{j i h}$.

(2) Grayan manifold. As S.Sasaki and Y.Hatakeyama [9 ], [14] proved, the Nijenhuis extensor $n_{\mu \lambda}{ }^{k}$ vanishes if and only if the tensor $n_{j i}{ }^{h}$ vanishes. have

(3) Almost semi-Sasakian manifold. Substituting (7.11) into (8. 1), we

$$
\left\{\begin{array}{l}
\nabla_{j} f_{i}{ }^{j}=(2 p-2) f_{i}, \\
\nabla_{j} f^{j}=-g^{j i} \nabla_{j} f_{i}=0 .
\end{array}\right.
$$

The first equation means that the fundamental 1 -form $\theta_{1}$ is coderived from the fundamental 2 -form $\theta_{2}$ :

$$
\theta_{1}=\frac{1}{2 p-2} \delta \theta_{2}
$$

and the second is satisfied automatically. Hence we have

THEOREM 8.2. An almost semi-Sasakian manifold is characterized by the property (8. 7) ${ }_{1}$ or (8. 8).

(4) Contact $O^{*}$-manifold. By use of $f_{i} f^{i}=-1$ and (7.10), the equation (8. 2) is separated into

$$
\left\{\begin{array}{l}
f_{j}^{a} \nabla_{a} f_{i}^{h}-f_{i}^{a} \nabla_{j} f_{a}{ }^{h}-f_{i} \nabla_{j} f^{h}-2 f_{j}^{h} f_{i}-2 f_{j i} f^{h}=0, \\
f_{j}^{a} \nabla_{a} f_{i}-f_{i}^{a} \nabla_{j} f_{a}-2 f_{j}^{a} f_{a i}=0, \\
f_{j}^{a} \nabla_{a} f^{h}-f^{a} \nabla_{j} f_{a}{ }^{h}+2 f_{j}^{a} f_{a}{ }^{h}=0, \\
f^{a} \nabla_{a} f_{i}^{h}=0 \\
f^{a} \nabla_{a} f^{h}=0 .
\end{array}\right.
$$

The second equation is equivalent to the third. The last two equations show that

THEOREM 8.3. In a contact $O^{*}$-manifold, the trajectories of the vector field $f^{h}$ are geodesic and the tensor field $f_{i}^{h}$ is parallel along the trajectories.

(5) Almost Sasakian manifold. Substituting (7. 12) into (8. 3), we have

$$
\begin{aligned}
& \nabla_{j} f_{i h}+\nabla_{i} f_{h j}+\nabla_{h} f_{j i}=0, \\
& \nabla_{j} f_{i}-f_{j i}-\nabla_{i} f_{j}+f_{i j}=0 .
\end{aligned}
$$

Since $f_{j i}$ is skew symmetric, the second equation yields to 


$$
f_{j i}=\frac{1}{2}\left(\nabla_{j} f_{i}-\nabla_{i} f_{j}\right)
$$

which means that the fundamental 2 -form $\theta_{2}$ is derived from the fundamental 1-form $\theta_{1}$ :

$$
\theta_{2}=d \theta_{1} \text {. }
$$

Therefore the first equation is satisfied automatically and we can say that

THEOREM 8.4. An almost Sasakian manifold is characterized by (8.10) or (8.11).

(6) Contact K-manifold. Substituting (7.12) into (8. 4), we have the equations

$$
\left\{\begin{array}{l}
\nabla_{j} f_{i h}-g_{j h} f_{i}+\nabla_{i} f_{j h}-g_{i h} f_{j}+2 g_{j i} f_{h}=0 \\
\nabla_{j} f_{i}+\nabla_{i} f_{j}=0 \\
\nabla_{j} f_{h}-f_{j h}=0 .
\end{array}\right.
$$

The second equation means that the vector field $f^{h}=-f_{i} g^{i h}$ is isometric. From the third, we have the equation (8.10). Thus we have

THEOREM 8.5. A contact K-manifold is an almost Sasakian manifold whose structure sasisfies the equations (8.12).

(7) Sasakian manifold. Substituting (7.12) into (8. 5), we have the equations

$$
\left\{\begin{array}{l}
\nabla_{j} f_{i h}=g_{j h} f_{i}-g_{j i} f_{h}, \\
\nabla_{j} f_{i}=f_{j i}
\end{array}\right.
$$

which characterize a Sasakian structure. Of course, a Sasakian structure has the properties of all the above mentioned structures.

9. Sasakian manifold. In a Kählerian manifold $\widetilde{M}$, we know well the identity

$$
\widetilde{K}_{\nu \mu \lambda}^{\alpha} F_{\alpha}{ }^{\kappa}-\widetilde{K}_{\nu \mu \alpha}{ }^{\kappa} F_{\lambda}{ }^{\alpha}=0 .
$$

Referring this equation to an adapted coordinate system and taking account of (7. 7), we have the identities ${ }^{(1)}$

$$
K_{k j i}^{a} f_{a}{ }^{h}-K_{k j a}{ }^{h} f_{i}^{a}=f_{k}^{h} g_{j i}-f_{j}^{h} g_{k i}-\delta_{k}^{h} f_{j i}+\delta_{j}^{h} f_{k i}
$$

and

(1) M. Okumura [13] obtained these identities from (8.13) by a direct computation. 


$$
K_{k j i}{ }^{h} f_{h}=f_{k} g_{j i}-f_{j} g_{k i} .
$$

In $\widetilde{M}$, we know also the identity

$$
\widetilde{K}_{\omega \kappa} F_{\mu}^{\omega} F_{\lambda}^{\kappa}=\widetilde{K}_{\mu \lambda},
$$

from which we obtain the identities

$$
\left\{\begin{array}{l}
K_{l k} f_{j}^{l} f_{i}{ }^{n}-(2 p-2) f_{j k} f_{i}{ }^{k}=K_{j i}-(2 p-2) g_{j i}, \\
K_{j i} f^{j} f^{i}=2 p-2 .
\end{array}\right.
$$

Putting

$$
\widetilde{H}_{\mu \lambda}=\widetilde{K}_{\mu}^{\kappa} F_{\kappa \lambda}=-\frac{1}{2} \widetilde{K}_{\mu \lambda \nu \kappa} F^{\nu \kappa},
$$

we know that $\widetilde{H}_{\mu \lambda}$ is skew symmetric and the form $\Xi$, defined by $\Xi=\widetilde{H}_{\mu \lambda} d x^{\mu}$ $\wedge d x^{\lambda}$, is closed. The induced from $\xi$ in $M$ is given by

$$
\xi=H_{j i} d y^{j} \wedge d y^{i}
$$

where

$$
H_{j i}=K_{j}^{k} f_{k i}
$$

The form $\xi$ is closed.

Since the vector field $f^{h}$ does not vanish, it follows from (9. 3) that

THEOREM 9.1 The local holonomy group of a Sasakian manifold is the full special orthogonal group $S O(2 p-1)$.

If the enveloping manifold $\widetilde{M}$ is of constant sectional curvature, i.e.

$$
\widetilde{K}_{\nu \mu \lambda \kappa}=\tilde{\kappa}\left(\delta_{\nu}^{\kappa} G_{\mu \lambda}-\delta_{\mu}^{\kappa} G_{v \lambda}\right)
$$

then we have $\widetilde{\kappa}=0$ because of $\widetilde{K}_{\infty j i}^{\infty}=0$ in $\widetilde{M}$, and $\widetilde{M}$ is locally euclidean. Hence, from (7. 7) and (7. 9), $M$ is of constant sectional curvature 1. The converse is clear. Thus we have

THEOREM 9.2. In order that the enveloping manifold $\tilde{M}$ of an almost Grayan manifold $M$ is of constant sectional curvature, it is necessary and sufficient that $M$ is of constant sectional curvatue $\kappa=1$, and then $\widetilde{M}$ is locally euclidean.

Similarly we have

THEOREM 9.3. In order that the enveloping manifold $\widetilde{M}$ is an Einstein manifold, it is necessary and sufficient that $M$ is an Einstein one of scalar 
curvature $\kappa=1$, and then $\widetilde{M}$ has zero Ricci curvature.

\section{REFERENCES}

[12] S. KотÔ, Some theorems on almost Kählerian spaces, J. Math. Soc. Japan, 12(1960), $422-433$.

[13] M. OKUMURA, Some remarks on space with a certain contact structure, Tôhoku Math. J., 14(1962), 135-145.

[14] S.SASAKI AND Y. HATAKEYAMA, On differentiable manifolds with contact metric structures, J. Math. Soc. Japan, 14(1962), 249-271. 